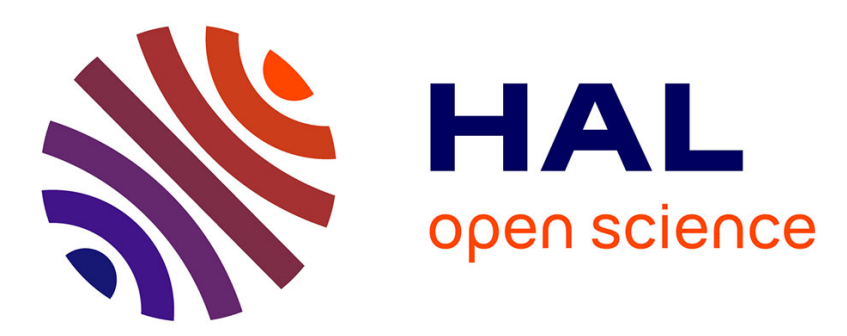

\title{
Relationship between local damage and macroscopic response of soft materials highly reinforced by monodispersed particles
}

Foucault de Francqueville, Pierre Gilormini, Julie Diani, Aude Vandenbroucke

\section{- To cite this version:}

Foucault de Francqueville, Pierre Gilormini, Julie Diani, Aude Vandenbroucke. Relationship between local damage and macroscopic response of soft materials highly reinforced by monodispersed particles. Mechanics of Materials, 2020, 146, pp.1-9. 10.1016/j.mechmat.2020.103408 . hal-02543517

\section{HAL Id: hal-02543517 https://hal.science/hal-02543517}

Submitted on 15 Apr 2020

HAL is a multi-disciplinary open access archive for the deposit and dissemination of scientific research documents, whether they are published or not. The documents may come from teaching and research institutions in France or abroad, or from public or private research centers.
L'archive ouverte pluridisciplinaire HAL, est destinée au dépôt et à la diffusion de documents scientifiques de niveau recherche, publiés ou non, émanant des établissements d'enseignement et de recherche français ou étrangers, des laboratoires publics ou privés. 


\title{
Relationship between local damage and macroscopic response of soft materials highly reinforced by monodispersed particles
}

\author{
Foucault de Francqueville",a,b, Pierre Gilormini ${ }^{\mathrm{c}}$, Julie Diani ${ }^{\mathrm{a}}$, Aude Vandenbroucke ${ }^{\mathrm{b}}$ \\ ${ }^{a}$ Laboratoire de Mécanique des Solides, CNRS UMR 7649, École Polytechnique, Institut Polytechnique de Paris, Route de Saclay, Palaiseau 91128, France \\ ${ }^{\mathrm{b}}$ ArianeGroup, Centre de recherche du Bouchet, 9 rue Lavoisier, Vert-le-Petit 91710, France \\ ${ }^{\mathrm{c}}$ PIMM, Arts et Métiers Institute of Technology, CNRS, Cnam, HESAM University, 151 boulevard de l'Hôpital, 75013 Paris (France)
}

\begin{abstract}
A B S T R A C T
A rubberlike matrix highly filled with spherical micrometric glass beads is submitted to uniaxial tension tests until break. X-ray tomography imaging performed on the material while submitted to uniaxial tension reveals early debonding at the matrix/filler interfaces at the poles of the particles followed by void coalescence creating damage localization. The latter causes a downturn of the macroscopic stress-strain response. These phenomena are analyzed further with three-dimensional finite element simulations, where 64 spherical beads are distributed randomly in a periodic cell. A simple version of the Tvergaard-Hutchinson cohesive-zone model allows to reproduce all the experimental trends well. The effects of the three parameters involved are analyzed, and three different types of macroscopic behaviors are observed corresponding to three different microstructure damages. The value of the initial stiffness of the interface, limited by numerical convergence, has little effect on how the local damage evolves but has a significant impact on the overall macroscopic stress values. The local damage is strongly dependent on the critical strength and the separation failure displacement, and the adhesion energy may be considered as a resulting parameter of the two previous ones. The interfacial critical strength appears to have a significant impact on the damage initiation, either spread across the structure for low values, or localized for high values. Increasing the interface separation failure displacement delays the possible loss of adhesion to a higher strain and preserves the integrity of the composite material.
\end{abstract}

\section{Introduction}

This contribution combines experimental observations and numerical simulations of the damage which develops at finite strain in soft materials reinforced by micron-size particles. While a material made of an acrylate soft matrix filled with sifted spherical glass beads was made in the lab for model reason, propellants are examples of such materials, where the material optimization translates into formulation changes that may weaken its strength and toughness because of an early appearance of cavities and cracks. The analysis of damage development at the scale of the reinforcements in relation with macroscopic loading may help optimizing the design of such composite materials.

The materials of interest are soft quasi-incompressible matrices capable of large reversible deformation and reinforced by a large amount (beyond 50\% volume fraction) of quasi-rigid particles. The large amount of fillers and of the strong stiffness contrast between the two constitutive phases increase the computational difficulties
(Segurado and Llorca, 2002). Since the polymer matrix is mostly submitted to monotonic loadings at temperatures well above the glass transition, viscoelasticity can be neglected. Recent studies have explored the nonlinear response of hyperelastic rubberlike matrix filled with rigid inclusions using three-dimensional computational homogenization with perfect filler/matrix adhesion (Lopez-Pamies et al., 2013; Guo et al., 2014; Leonard et al., 2020). In propellants as in other elastomers filled with micron size particles, it is common to observe matrix debonding at the filler surface (Cornwell and Schapery, 1975; Oberth and Bruenner, 1965; Li et al., 2018), which demands to account for a damageable adhesion at the matrix/filler interface. For this purpose, cohesive-zone models (Tvergaard and Hutchinson, 1993; Park et al., 2009) have already been introduced in finite element simulations involving spherical particles dispersed in a matrix (Segurado and Llorca, 2005; Matouš and Geubelle, 2006; Spring and Paulino, 2015; Cho et al., 2017; Gilormini et al., 2017). The account for matrix adhesion damage has shown to produce a realistic macroscopic behavior

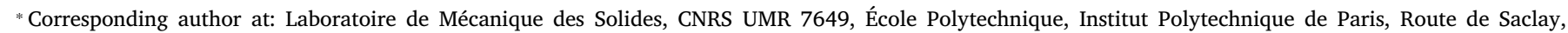
Palaiseau 91128, France.

E-mail address: foucault.de-francqueville@polytechnique.edu (F. de Francqueville). 
that could not be reproduced otherwise (Inglis et al., 2007). Early studies (Böhm et al., 2004; Llorca and Segurado, 2004; Segurado and Llorca, 2005, among others) were focusing on ductile matrices with infinitesimal strain formalism. More recently, account for composites with hyperelastic matrices, for which softening may result from the matrix/filler interface damage only, were considered in finite strain in two-dimensional (Moraleda et al., 2009; Toulemonde et al., 2016; Zhang et al., 2018; Li et al., 2018) and three-dimensional representations (Spring and Paulino, 2015; Gilormini et al., 2017). While some of these contributions have focused on the impact of the cohesive zone model parameters on the macroscopic behavior (Spring and Paulino, 2015; Toulemonde et al., 2016), others have looked at the distribution of stresses in the matrix (Moraleda et al., 2009; Li et al., 2018). The link between the local damage field and the macroscopic stress-strain response remains to be examined and we propose to do so by using a three-dimensional computational homogenization approach.

In this paper, a close look at the local damage as a function of the material parameters and the microstructure will help unveiling the phenomena that induce the features exhibited by the macroscopic behavior. It was noted that according to the cohesive zone model parameters featuring different adhesion properties, three different types of macroscopic behaviors could be witnessed with the same microstructure, resulting from three different types of local microscopic damage. The numerical simulations will consider three-dimensional periodic cells, where mono-size spherical particles are embedded in a soft matrix with a Tvergaard-Hutchinson cohesive-zone model used at the matrix/filler interfaces. Actually, previous two-dimensional studies (Matouš et al., 2007; Toulemonde et al., 2015) have shown the minor interest of considering polydisperse filler sizes in our case, since damage concentrates first and foremost around the largest particles. The macroscopic behavior as well as the local damage obtained on the numerical material will be discussed in the light of original lab measurements and observations collected on an actual rubbery acrylate matrix filled with sifted glass beads. The paper is organized as follows. In Section 2, an experimental characterization of the macroscopic behavior and local observations of damage obtained on the actual reference material are reported. The numerical simulation framework is detailed in Section 3, highlighting the cohesive-zone model representing the damageable adhesion of the matrix/particle interfaces. Finally, Section 4 presents an analysis of the impact of the parameters of the cohesive-zone model on the local damage and resulting composite macroscopic stress-strain responses with an attempt to provide with a quantitative comparison between the model and the experiments. Concluding remarks close the paper.

\section{Mechanical behavior and local damage of an actual model material}

\subsection{Reference material}

A rubbery polymer matrix network has been highly filled with 200/ $250 \mu \mathrm{m}$-sifted glass beads. The matrix was obtained by mixing $98 \%$ molar mass of methacrylate (MA) and $2 \%$ molar mass of polyethylene glycol dimethacrylate (PEGDMA) of molar weight $750 \mathrm{~g} / \mathrm{mol}$, with photo-initiator 2,2-Dimethoxy-2-phenylacetophenone (DMPA). The products were used as received by Sigma Aldrich. The glass beads were first cleaned following Cras et al. (1999) recommendations and slowly added to the stirring mix. The pasty blend was poured in a $3 \mathrm{~mm}$-thick transparent mold and exposed to UV light during 40 minutes for polymer crosslinking. Once removed from the mold, the composite plates were subjected to a temperature of $45{ }^{\circ} \mathrm{C}$ during 15 minutes for annealing possible process residual stresses.

Samples of final dimension $40 \times 10 \times 3 \mathrm{~mm}^{3}$, as displayed in Fig. 1a, were punched in the plates. For each sample, the glass bead volume fraction was estimated accurately with an AccuPycII 1340 gas pycnometer. Finally, samples showing the targeted 55\% volume fraction of glass beads were selected. The three-dimensional material microstructure was characterized by microtomography X-ray scans (XR$\mu \mathrm{CT}$ ) as illustrated in Fig. 1c. The random dispersion of the glass beads was partially assessed (de Francqueville et al. (2019) )by comparing the two-point correlation function $S_{2}$ (Torquato, 2002) computed from the reference material microstructure and the values calculated for a Percus-Yevick distribution for the same volume fraction (Katzav et al., 2017).

\subsection{Stress-strain response in uniaxial tension}

For mechanical testing, samples were glued (with Loctite Flexcomet PU 15) to aluminum jaws and uniaxial tensile tests were carried out at the low constant crosshead speed of $0.1 \mathrm{~mm} / \mathrm{min}$ on an Instron 5967 tensile machine. The strain was measured by video extensometry based on a Labview routine following two dots recorded by a 3 MPx camera equipped with a telecentric lens. Fig. 2 illustrates the stress-strain responses recorded on three samples. Beyond the good reproducibility of the test, it is worth noting that the behavior of the material presents four stages. First, at small strain, the material response is linear (stage 1). Then, the material stress-strain slope decreases (stage 2), a sign of the appearance of damage since the matrix is a rubbery amorphous polymer and does not exhibit any plasticity. Around 7\% strain, a downturn occurs (stage 3) followed by a final upturn (stage 4) before the sample ruptures. Note that the final strain at break is rather scattered, which might be due to local defaults induced when making or cutting the samples. For this type of composite, the damage analysis is usually handled by a measurement of the global volume change (Farris, 1968), which cannot help understanding the difference of behavior between stages 2 and 3, whereas tomography observations performed on stretched samples give more insights on the local damage attached to each stage of the mechanical behavior.

\subsection{Microscale damage upon uniaxial tensile loading}

The aim of the experiments is to study the evolution of the damage at the matrix/filler interface at various tensile loading levels by means of XR- $\mu \mathrm{CT}$ imaging. The images were captured with the laboratory scanner available at Laboratoire Navier (Ultratom from RX-Solution) providing here a resolution of $5 \mu \mathrm{m}$ per voxel. In order to scan samples at different stretch levels without adapting a specific setup to the scanner, the samples were first stretched on the Instron machine and then glued on glassy acrylate plates which maintained the deformation state. Doing so, an undeformed sample as well as three samples stretched at 5,10 and $15 \%$ strain respectively could be scanned.

Fig. 3 shows representative slices for the four samples. In the initial state, only particles (white) and matrix (light gray) are clearly visible. Very few voids (black) at matrix/filler interfaces could be detected, which were probably induced when punching the samples. At $5 \%$ strain, several voids are evidenced at matrix/filler interfaces, at either one or both poles of the debonded particles. At $10 \%$ strain, some voids coalesced and formed micro cracks, which may be regarded as a damage localization and is consistent with the downturn observed on the macroscopic stress-strain curve at this strain level during the uniaxial tensile tests. When the material is close to failure (15\% strain), the size of the micro cracks seems reasonably unchanged but their number is much larger. Therefore, the first softening characterized by stage 2 in Fig. 2 is related to a matrix debonding that is homogeneously dispersed in the material, then the appearance of micro cracks is responsible for the downturn of the stress-strain curve corresponding to stage 3 of the material stress-strain response. The final upturn before break is likely due to the stiffening response of the matrix when submitted to larger strain in the regions where it provides the main contribution to the response of the damaged composite. On a quantitative side, using the mean value of the scans gray level, it was possible to quantify the volume fractions of void to $6.3 \% \pm 1.3 \%$ for the sample strained at $10 \%$ 


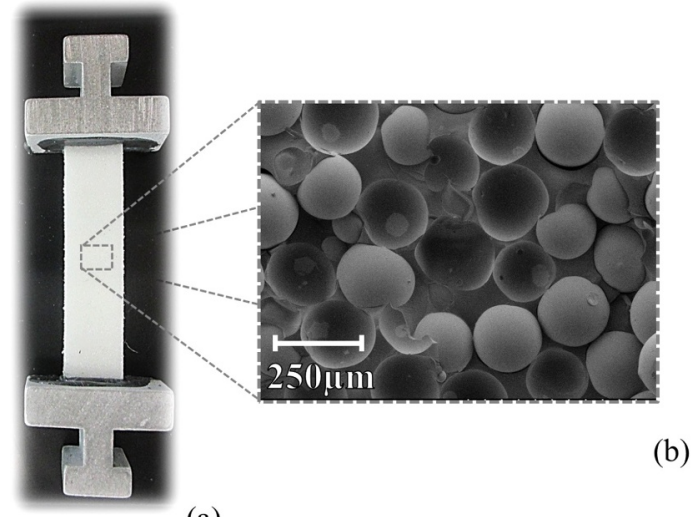

(a)

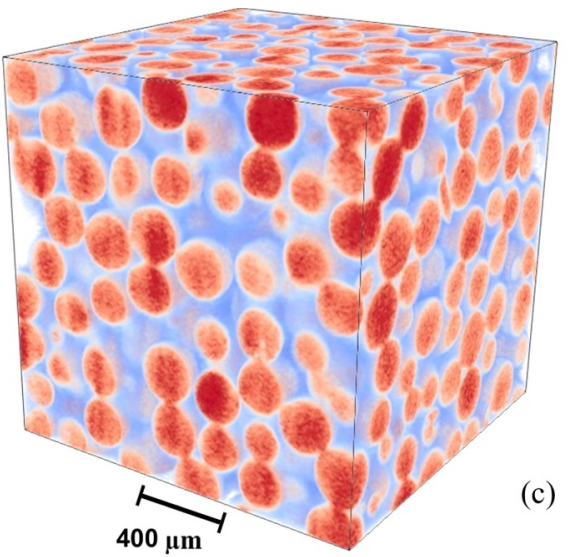

Glass

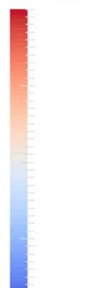

Elastomer

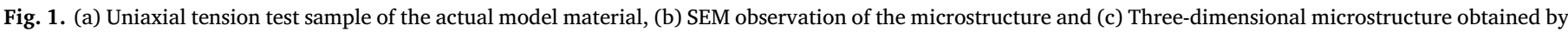
$\mathrm{X}$-Ray tomography. The composite is made of a $55 \%$ volume fraction of glass beads dispersed randomly in a rubbery acrylate matrix.
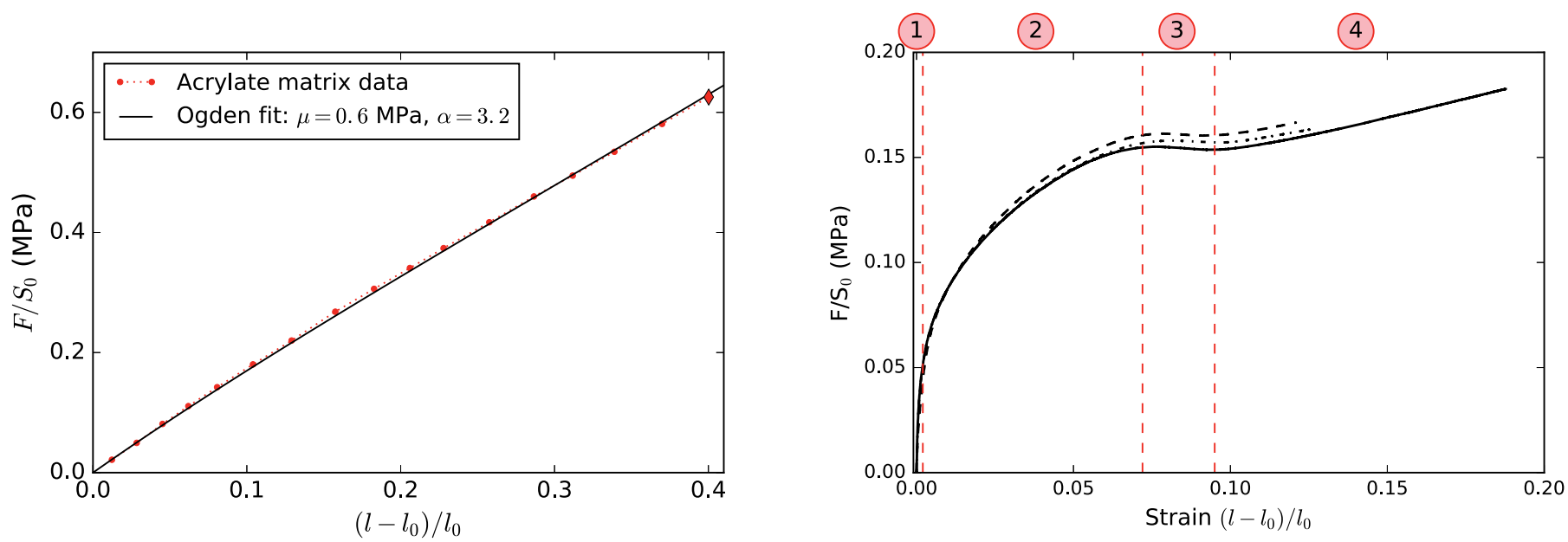

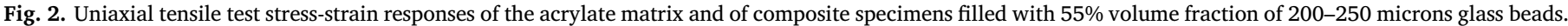

and $12.2 \% \quad \pm 1.2 \%$ for the sample strained at $15 \%$. unfortunately, the uncertainty was too large to provide with a reliable quantitative value for the sample strained at $5 \%$. These microstructural observations show the impact of the local damage in the material response, and the purpose of the next section is to set the numerical simulations that will help studying the impact of the adhesion parameters on the composite damage at the micro scale and on its macroscopic behavior.

\section{Numerical material}

\subsection{Microstructure generation and numerical framework}

The micromechanical simulations require to generate and select representative microstructures and to account for mechanical properties similar to the reference actual material. Microstructures were generated using the Skoge et al. (2006) method based on the Lubachevsky and Stillinger (1990) algorithm, which provided isotropic periodic cells starting from 64 randomly dispersed particle seeds

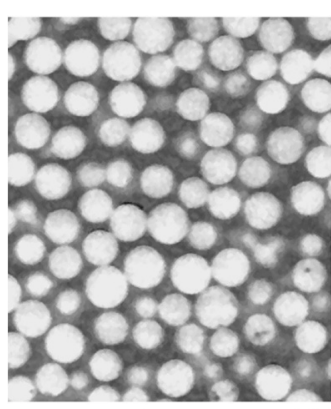

(a)

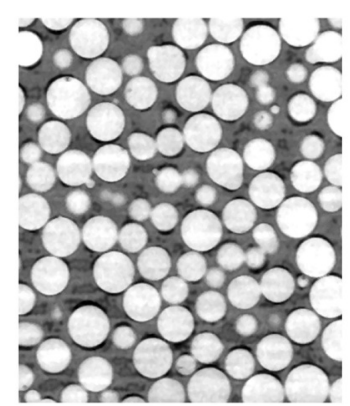

(b)

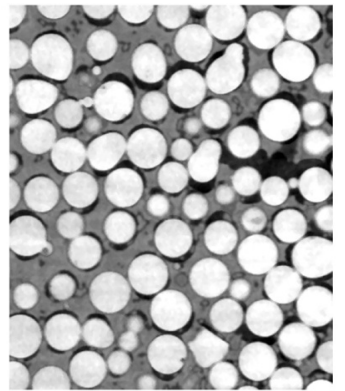

(c)

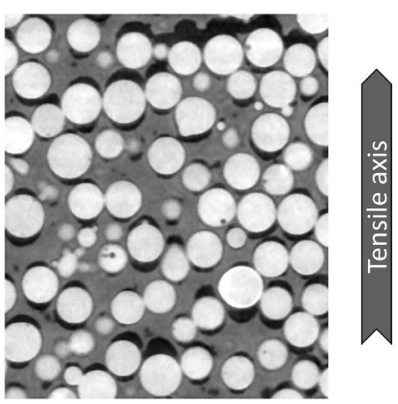

(d)

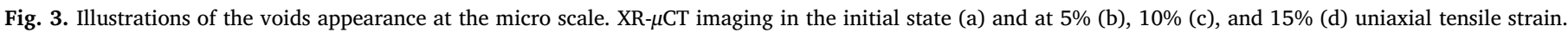



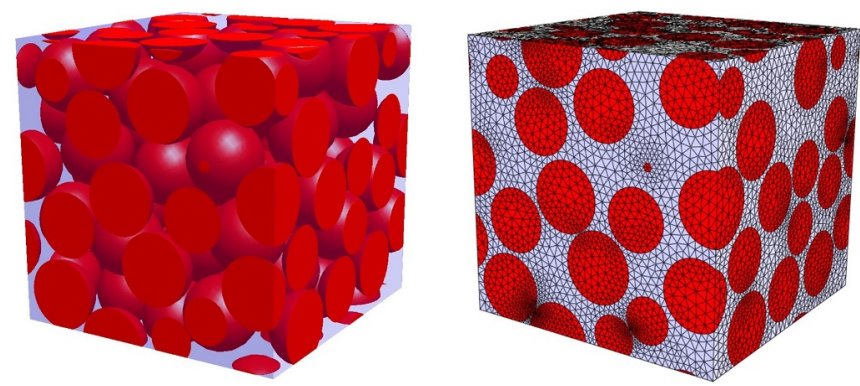

Fig. 4. Example of a generated periodic microstructure with 64 single-size particles at 55\% volume fraction before and after finite element meshing with noticeable element refinement in the areas of near-to-touch particles.

(de Francqueville et al., 2019). Finite element analyses have been carried out using Abaqus (2018) finite element code, for is its proven ability to cope with large strain and with interface debonding through cohesive-zone models (Park and Paulino, 2011). First, a conforming periodic mesh of ten-node quadratic tetrahedra was obtained with the Netgen free software (Schneider et al., 2016), which is able to list the displacement constraints required to apply periodic boundary conditions. This process included mesh refinements in areas of near-to-touch spheres to avoid element distortions. Note that no minimum distance between beads was applied, following the recommendations of Gusev (2016). The number of elements was varied from 500,000 to 2 millions in order to assess the mesh convergence that has been evaluated with the Young modulus at infinitesimal strain. Fig. 4 shows an example of a periodic microstructure before and after finite element meshing. After this meshing process, each node located at the surface of the fillers was duplicated and, if located also on a cell boundary, the associated displacement constraints to comply with periodic boundary conditions were listed. This allowed to define 12-node prismatic user elements with an initial zero thickness, where the cohesive-zone model could be applied. Since no direct visualisation was available for user elements, dummy six-node triangular surface elements were also defined at the surface of the fillers, to which any value computed in the corresponding user elements could be copied through a UVARM user routine. This procedure was used to display the damage level at each matrix/filler interface.

The beads behavior was defined by typical elastic parameters for glass with a Young modulus of $69 \mathrm{GPa}$ and a Poisson's ratio of 0.25 . The hyperelastic behavior of the matrix was measured in uniaxial tension, and the Ogden strain energy density (Ogden, 1982) appeared to fit the matrix response well with a single set of Ogden parameters $\mu=0.6 \mathrm{MPa}$ and $\alpha=3.2$ (Fig. 2). Since rubberlike materials are quasi-incompressible, with a bulk modulus three orders of magnitude larger than their shear modulus, the compressibility factor of the Ogden law was defined as $D=6.6667 .10^{-4} \mathrm{MPa}^{-1}$, corresponding to a bulk modulus of $3 \mathrm{GPa}$ and to a Poisson's ratio of 0.4999 . Therefore, Abaqus $\mathrm{C} 3 \mathrm{D} 10 \mathrm{H}$ hybrid elements were used in the matrix phase. In order to neglect the impact of the dummy surface elements on the overall stiffness, their mechanical properties were defined as elastic with a negligible Young modulus of $10^{-20} \mathrm{MPa}$ and a Poisson's ratio of 0.25 .

Finally, a typical mesh was made of about one million nodes and half a million elements, with three millions degrees of freedom. The simulation of a uniaxial tensile test lasted between 12 and $36 \mathrm{~h}$ when run in parallel on 10 cluster nodes with $2.5 \mathrm{GHz}$ Intel Xeon E5-2640 CPUs and 64 GB RAM for each node.

\subsection{Cohesive-zone model}

A simple version of the traction-separation law proposed by Tvergaard and Hutchinson (1993) has been selected for its small number of parameters when normal and tangent separation laws are assumed similar. An illustration of this law in the case of purely normal

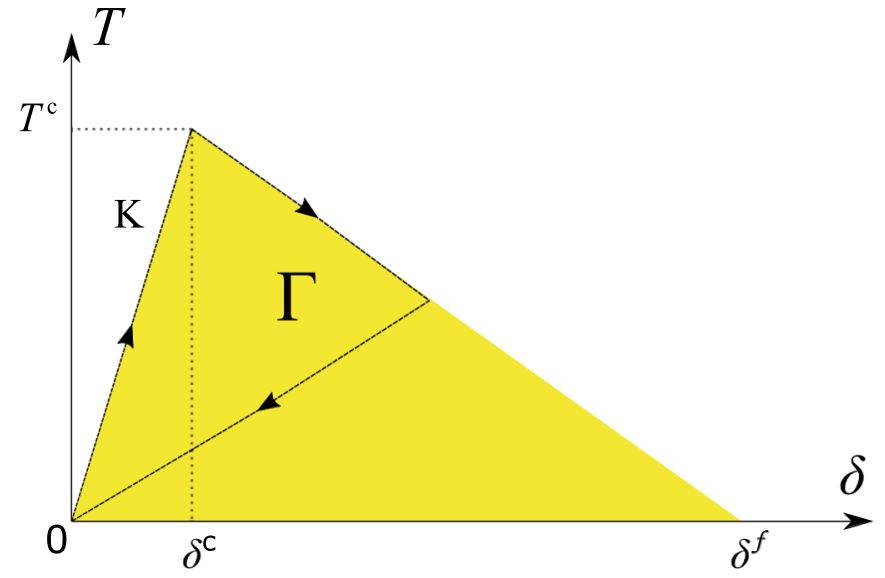

Fig. 5. Traction-separation behavior of a cohesive element for a purely normal (or purely tangent) displacement defined by three independent parameters among the five $\left(K, \Gamma, T^{c}, \delta^{c}, \delta^{f}\right)$ displayed here.

(or purely tangent) displacement is presented in Fig. 5, where the notations are defined. The non-dimensional separation factor $\lambda$ is introduced, such that the cohesive zone is ruptured when $\lambda=1$ in pure or mixed loading mode:

$\lambda=\sqrt{\left(\frac{\left\langle\delta_{n}\right\rangle}{\delta^{f}}\right)^{2}+\left(\frac{\delta_{t}}{\delta^{f}}\right)^{2}}$

with $\delta_{n}$ and $\delta_{t}$ denoting the normal and tangent displacements of the interface, respectively. Note that the operator $\langle\bullet\rangle$, which stands for Macaulay brackets, allows the distinction between normal separation and compression, with the latter inducing no change of the cohesive stiffness. The largest separation factor encountered at the interface is stored as $\lambda^{m}(t)=\max _{t} \lambda(t)$ in order to keep track of the evolving properties of adhesion. This parameter is related directly to the damage factor $D$, which is equal to 0 when the separation displacement is lower than the critical displacement $\delta^{c}$ and increases up to 1 when the displacement at failure $\delta^{f}$ is reached and $\lambda=1$ :

$D=\frac{1}{\lambda^{m}}\left(\frac{\left\langle\lambda^{m}-\frac{\delta^{c}}{\delta^{f}}\right\rangle}{1-\frac{\delta^{c}}{\delta^{f}}}\right)$.

With this definition, the normal and tangent stresses write as

$\begin{cases}T_{n}=K \delta_{n} & \text { if } \delta_{n}<0 \text { (compression) } \\ T_{n}=K\langle 1-D\rangle \delta_{n} & \text { if } \delta_{n}>0 \\ T_{t}=K\langle 1-D\rangle \delta_{t} & \end{cases}$

Once noting the relations (see Fig. 5)

$\delta^{c}=\frac{T^{c}}{K} \quad$ and $\quad \Gamma=\frac{T^{c} \delta^{f}}{2}$,

only three independent parameters remain to define the cohesive law, for instance the initial stiffness $K$, the critical strength $T^{c}$ and the displacement at failure $\delta^{f}$. Classically, attention is rather focused on the adhesion energy $\Gamma$, which is directly related to the physics of adhesion. However there is no obvious way to estimate this property at the scale of the particles precisely (see Tan et al., 2005 or Gilormini et al., 2017, for attempts to estimate the cohesive parameters experimentally). Therefore, the displacement at failure $\delta^{f}$ has been preferred, whose order of magnitude can be compared to the dimensions of the particles. According to the experimental observations reported in Fig. 3, complete debonding should be possible before the maximum macroscopic strain is reached, which will limit the value of $\delta^{f}$ to the order of one tenth of 


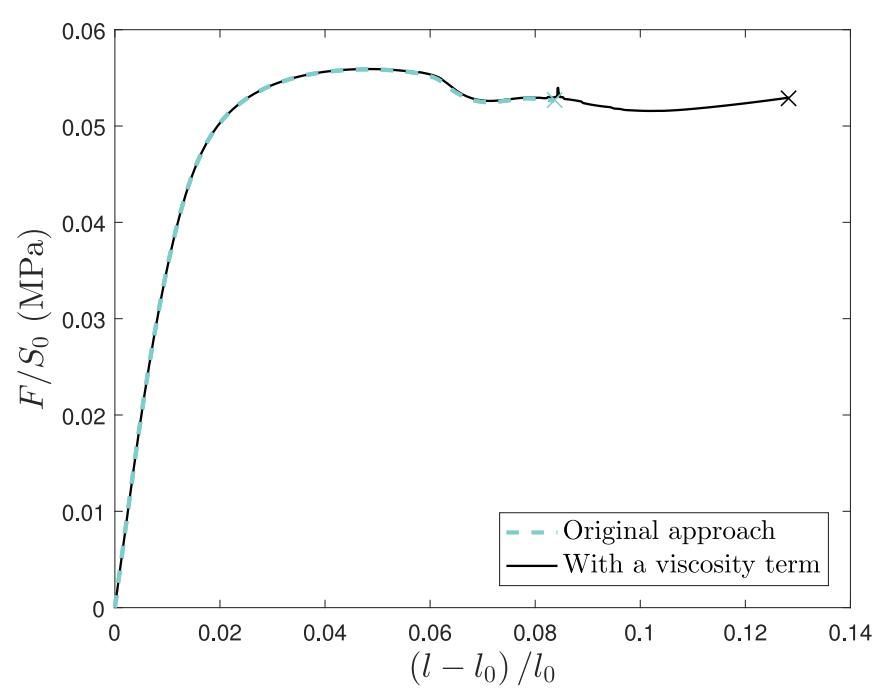

Fig. 6. Comparison of the stress-strain responses of a numerical microstructure submitted to a uniaxial tensile test when including or not including a fictitious viscosity in the Tvergaard-Hutchinson separation-traction law at the matrix/ filler interfaces. Numerical divergence is marked by a cross.

the spheres radius.

The Tvergaard and Hutchinson cohesive-zone model is available in Abaqus for linear elements only, and therefore a user subroutine UEL has been written in order to implement this model in a 12-node interface quadratic user element. Moreover, this implementation included a fictitious viscosity $\zeta$ to improve numerical convergence. Adapting the technique of Gao and Bower, (2004) technique, Eq. (3) is modified into

$$
\begin{cases}T_{n}=K \delta_{n} & \text { if } \delta_{n}<0 \text { (compression) } \\ T_{n}=K\langle 1-D\rangle \delta_{n}+\zeta_{n} \frac{\mathrm{d}}{\mathrm{d} t}\left(\frac{\delta_{n}}{\delta^{f}}\right) & \text { if } \delta_{n}>0 \\ T_{t}=K\langle 1-D\rangle \delta_{t}+\zeta_{t} \frac{\mathrm{d}}{\mathrm{d} t}\left(\frac{\delta_{t}}{\delta^{f}}\right) & \end{cases}
$$

Viscous regularization is commonly used to improve convergence (Chaboche et al., 2001; Hamitouche et al., 2008) however, the viscous parameter should not modify the mechanical response or induce unreasonable computational cost. Based on Gao and Bower (2004), the fictitious viscosity $\zeta$ has been estimated as one thousandth of the adhesion energy divided by a typical velocity applied to the cell boundary during the tensile test. This allowed to extend the applied strain at divergence significantly, while the impacts on the macroscopic behavior in uniaxial tension and on the local damage were negligible. Fig. 6 shows the macroscopic stress-strain responses with and without viscosity coinciding up to divergence of the former. This example has been chosen for the particularly large strain value reached without viscosity, allowing a representative comparison when numerous interface elements are fully damaged.

\subsection{Computational analysis}

The macroscopic stress-strain response can now be discussed in the light of the field of the separation factor $\lambda$ for a series of numerical simulations defined by a given microstructure and by a set of cohesivezone parameters. The parameter $K$, defining the initial stiffness of the cohesive elements, has a strong impact on the initial linear elastic response of the composite material but, when large enough, it does not affect significantly the strain at which softening initiates or the macroscopic stress-strain response beyond the linear initial response. However, $K$ has a dramatic impact on the numerical convergence, which excludes extremely stiff interface elements. A constant value of $K=70 \mathrm{MPa} / \mathrm{mm}$ has proven to provide with a good compromise

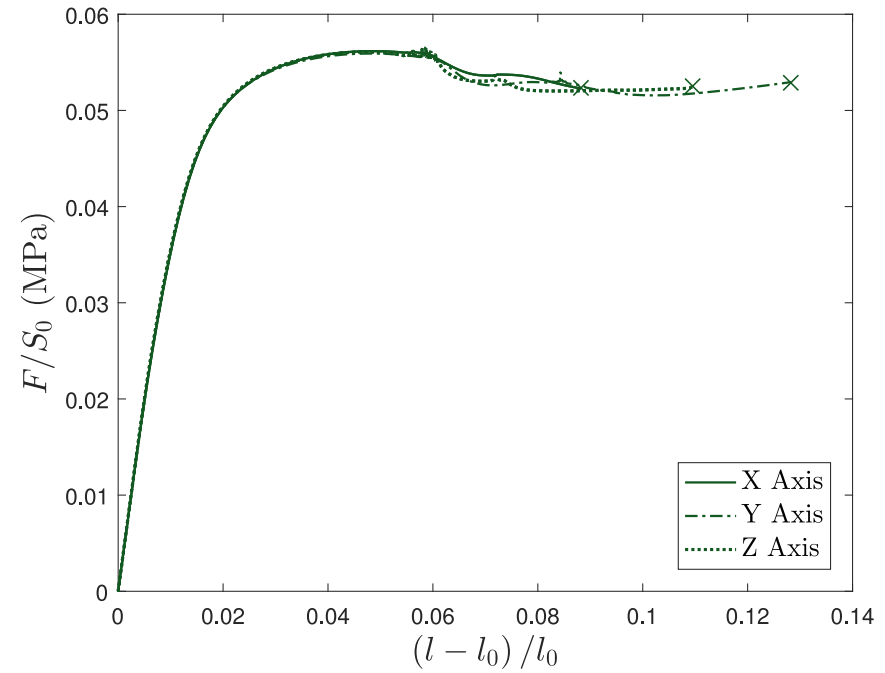

Fig. 7. Stress-strain responses of a microstructure submitted to uniaxial tensile loading along each Cartesian axis with the same material parameters. Computational divergence is indicated by cross symbols.

allowing changes of values of $T^{c}$ and $\delta^{f}$, and therefore to study the impacts of the traction-separation law on the macroscopic mechanical response and on the occurrence of local damage. It is worth noting that results will focus on how, where and when damage will appear in the microstructure. Indeed, due to the periodic boundary conditions used here, which were required to reduce the size of the numerical problem to a reasonable extent, once localization appears it may self-sustain.

Finally, the isotropy of the generated microstructure at large strain was evaluated by running the same uniaxial tensile loading along each Cartesian axis of the cubic periodic box. Fig. 7 shows three similar numerical stress-strain responses allowing us to run simulations in one directions only in what follows.

\section{Results and discussion}

\subsection{Impact of the interfacial behavior}

For a given microstructure, various macroscopic stress-strain responses can be obtained according to the properties of the cohesive zone. In order to illustrate the different types of stress-strain responses and look at the corresponding local damage, three sets of cohesive zone parameters, listed in Table 1, are considered. The first case considers a small value of $T^{c}$, allowing the damage to appear early and develop across the microstructure. It also accounts for a realistic value $\delta^{f}$ of 20 microns compared to the spheres radius. The second case presents a larger value of $T^{c}$, evaluating the impact of an increase in the interface strength. The last case looks at the effect of a larger displacement at failure $\delta^{f}$ compared to the second case, testing the increase of the interface toughness.

Fig. 8 shows the macroscopic stress-strain responses obtained for the three sets of cohesive zone parameters. The behaviors start similarly with a linear response whose slope depends on the stiffnesses of the constitutive phases. Then, the material responses soften due to the appearance of matrix/particle interfacial damage. In case 1 of low

Table 1

Parameters of the cohesive zone model (Fig. 5).

\begin{tabular}{llll}
\hline & $K(\mathrm{MPa} / \mathrm{mm})$ & $T^{c}(\mathrm{MPa})$ & $\delta^{f}(\mathrm{~mm})$ \\
\hline Case 1 & 70 & 0.05 & 0.02 \\
Case 2 & 70 & 0.1 & 0.02 \\
Case 3 & 70 & 0.1 & 0.06 \\
\hline
\end{tabular}




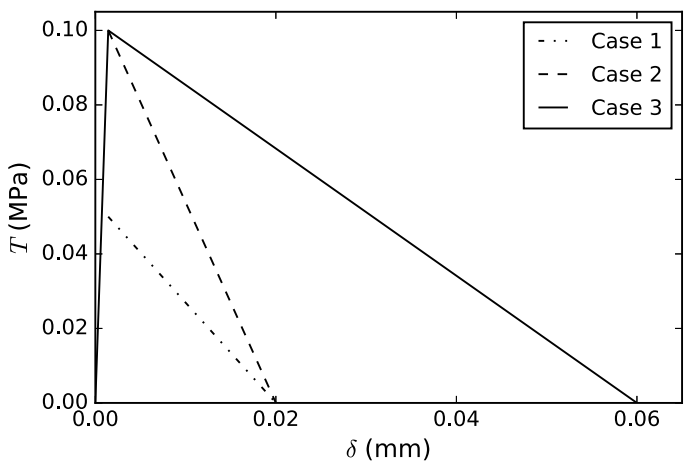

(a)

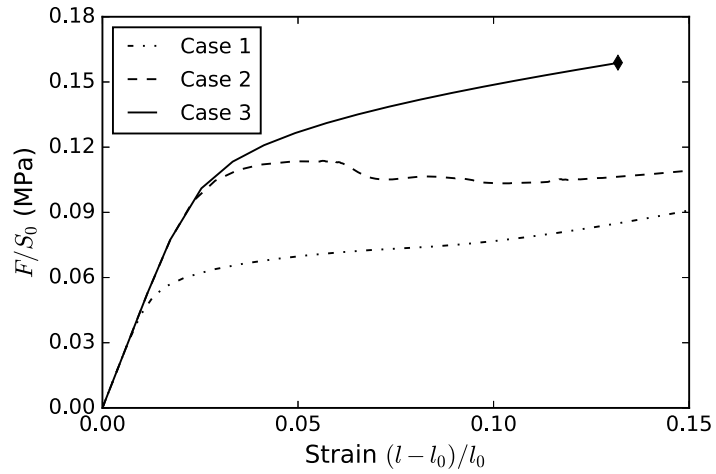

(b)

Fig. 8. Impact of the cohesive zone parameters on the macroscopic stress-strain response. (a) Illustration of the considered traction-separation behaviors. (b) Macroscopic stress-strain responses. Symbols designate computational divergences.

$3 \%$ strain
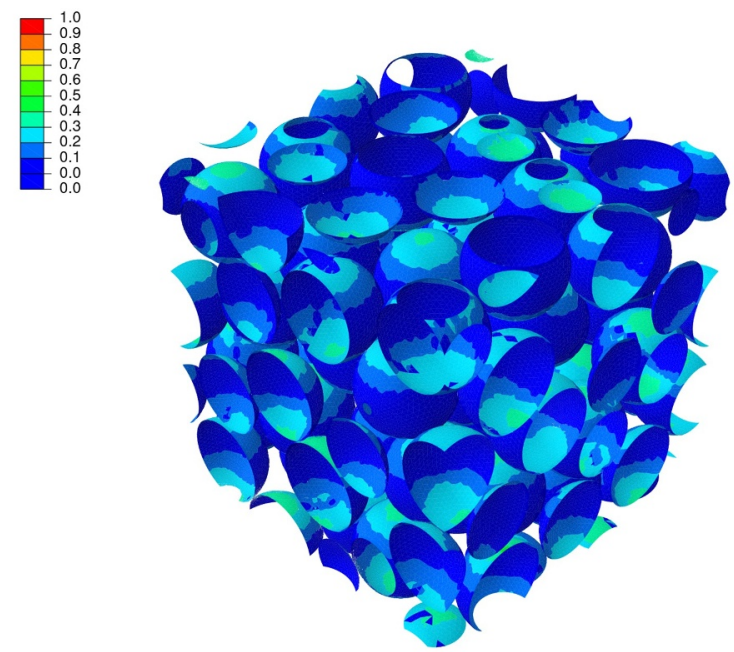

$7 \%$ strain
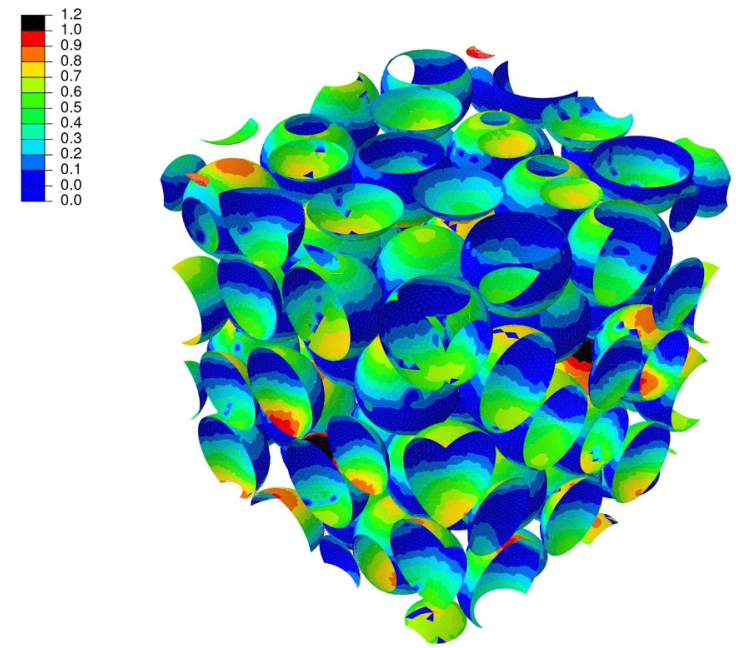

$5 \%$ strain
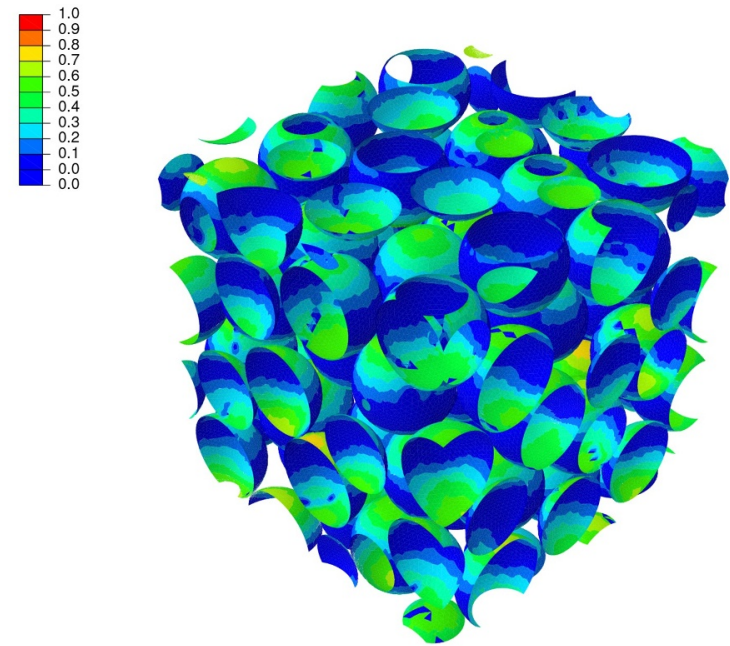

$10 \%$ strain
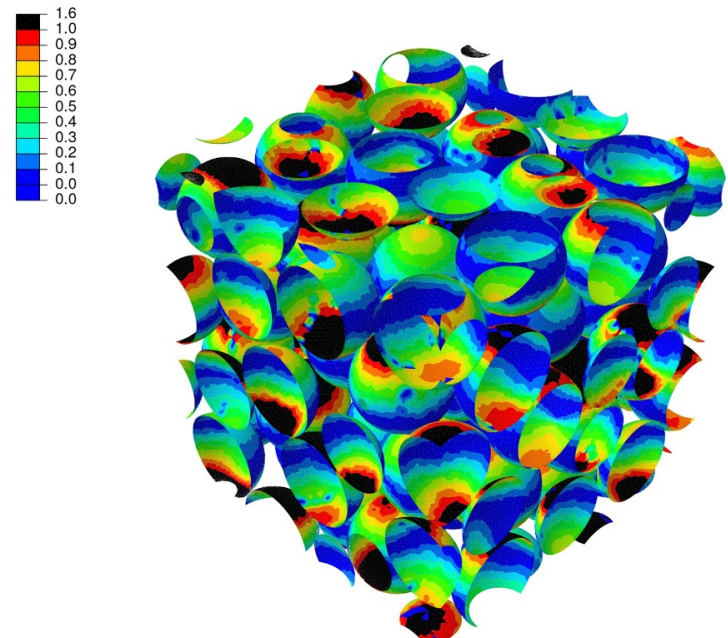

Fig. 9. Illustration of the separation factor $\lambda$ recorded at 3, 5, 7 and 10\% macroscopic strain during vertical tensile tests resulting in Case 1 macroscopic stress-strain behavior displayed in Fig. 8 . 

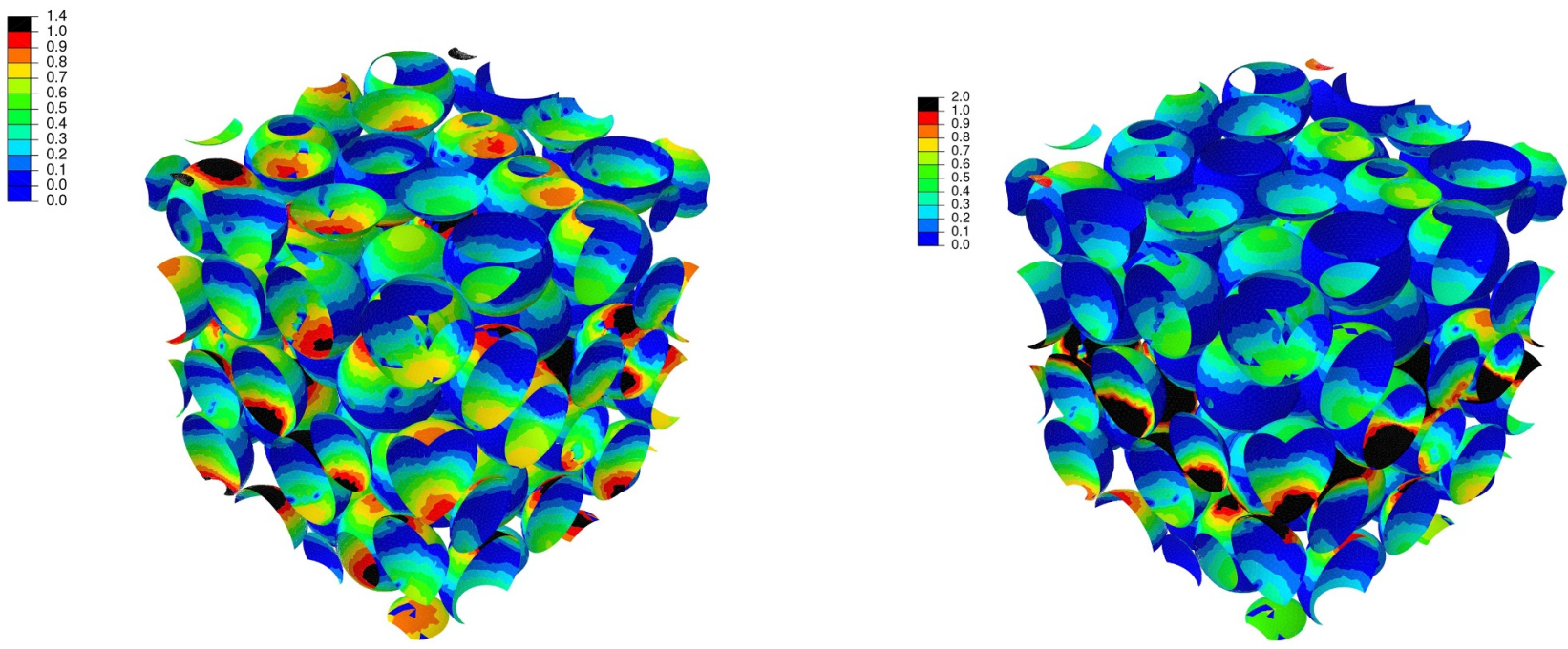

Case 3
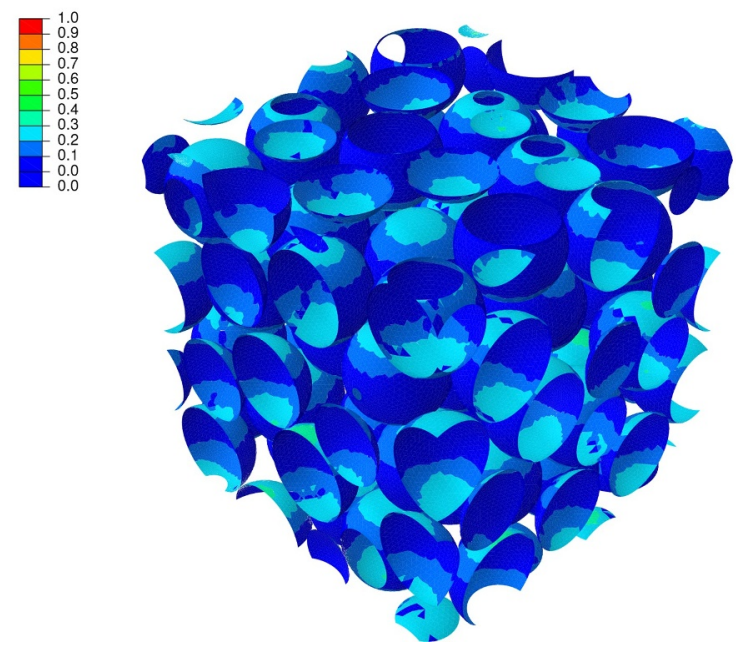

Fig. 10. Illustration of the separation factor $\lambda$ recorded at $8 \%$ macroscopic strain during vertical tensile tests resulting in the macroscopic stress-strain behaviors displayed in Fig. 8.

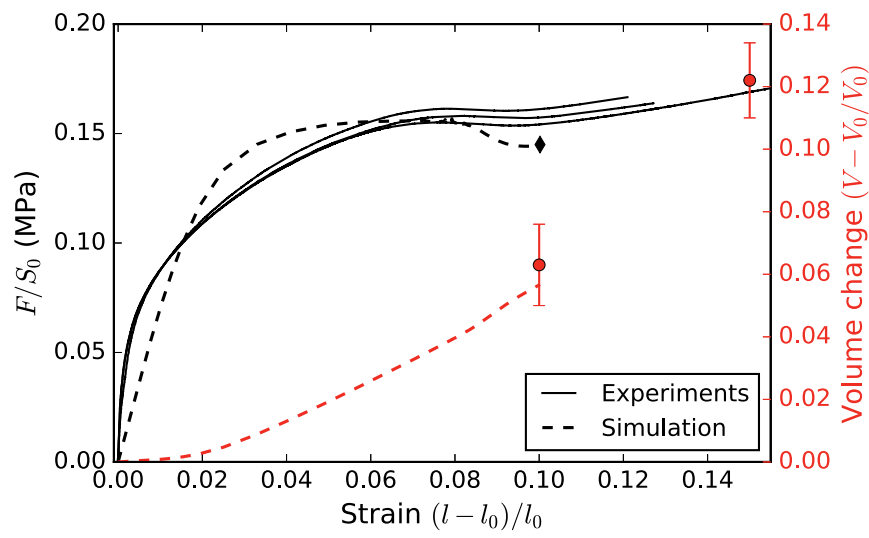

Fig. 11. Quantitative comparison between the numerical model and the experiments. interfacial strength, the stress at which damage initiates is low. The stress-strain response evolves quasi-linearly after damage starts. In case 2 , the interfacial strength has been increased, keeping the displacement at failure $\delta^{f}$ constant. As one could expect, increasing the interfacial strength enhances the macroscopic strength. More interestingly, the stress-strain response shows a downturn in the same fashion as the one witnessed on the actual material Fig. 2. Finally, in case 3, the interfacial toughness has been extended slowing down the evolution of stiffness loss at the interface. Like in case 1, the stress-strain response evolves quasi-linearly after the damage sets in. The stress-strain curves showed in Fig. 8, are not only different in terms of values but also in terms of shapes and one shape exhibits the same trends as the actual materials (Fig. 2). In order to better understand these numerical results, a closer look is taken at how the microscopic damage appears and evolves in the microstructure.

First, a close look at the appearance and evolution of damage around the particles is taken for the simulation Case 1. Fig. 9 presents the separation factor at the matrix/beads interface at 3, 5, 7 and 10\% macroscopic vertical uniaxial strain. Damage appears (3\% strain) and 


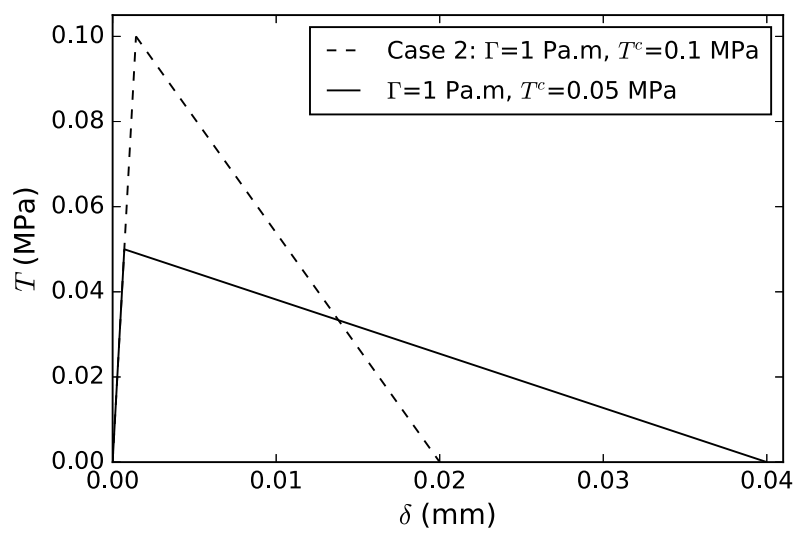

(a)

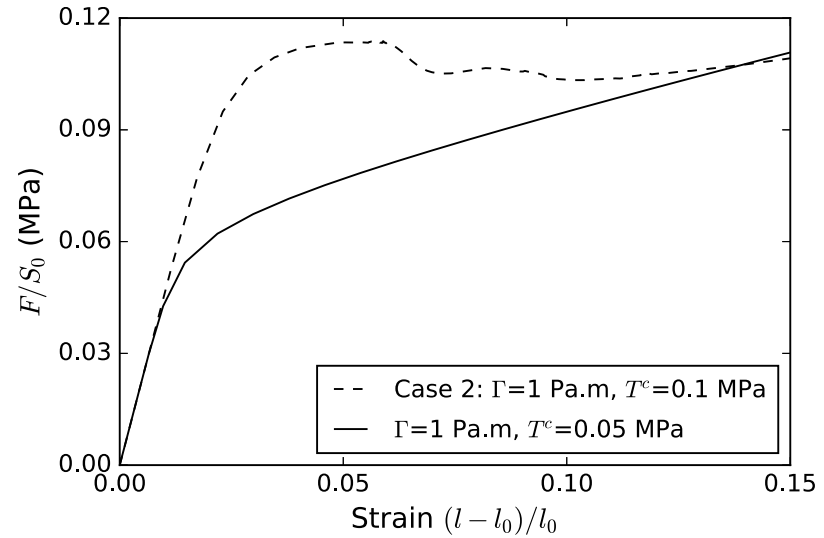

(b)

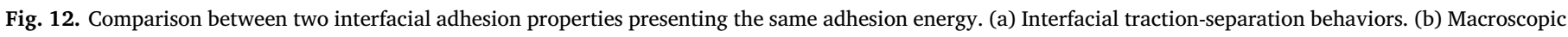
stress-strain responses.

develops (5\% strain) evenly at both poles of numerous particles aligned with the tensile direction. Then, it increases faster at one pole, relaxing the separation at the other pole ( $7 \%$ strain). Finally, when complete separation is reached at the mostly damaged pole, damage increases again at the other pole (10\%).

Second, Fig. 10 compares the local separation factor recorded at the matrix/filler interfaces at $8 \%$ macroscopic strain for the stress-strain responses shown in Fig. 8. Case 1 shows evenly scattered significant damage across the microstructure, which was observed as typical of a low value of critical strength $T^{c}$. Some elements have reached the complete failure $(\lambda \geq 1)$. Case 2 presents severe damage localized in a plane perpendicular to the vertical tension. The fact that damage develops preferentially in a plane perpendicular to the tension induces the macroscopic specific stress-strain response displaying a downturn. It results from the combination of a large $T^{c}$ and a small $\delta^{f}$, the first parameter causing damage to appear preferentially on certain particles while the second parameter leads to fast damage evolution before damage could spread to other particles. This particular case is in accordance with the macroscopic and microscopic characteristics of the actual material. Finally, Case 3 shows limited damage evenly scattered across the microstructure. Due to the large separation failure, once damage appears on a few particles it evolves slowly favoring its appearance on other particles.

The simulations have provided with a better understanding on how damage takes place and evolves according to the interfacial properties. The critical strength $T^{c}$ and separation factor $\delta^{f}$ appear to have key roles on the local microscopic damage that drives the macroscopic stressstrain response. Moreover, some clues have emerged on the interfacial properties of the actual material, which could be characterized by a large critical strength and a small separation at failure. Next, the capability and the limitation of our simple numerical model is discussed at the light of an attempt for quantitative comparison between the simulations and the experimental data.

\subsection{An attempt to reproduce the experimental data}

Fig. 11 presents a comparison between the numerical model and the experiments. The theoretical result was obtained for the following cohesive zone parameters: $K=200 \mathrm{MPa} / \mathrm{mm}, T^{c}=0.14 \mathrm{MPa}$ and $\delta^{f}=0.027 \mathrm{~mm}$ (or equivalently $\Gamma=1.9$ Pa.m). On the upside, the order of magnitude of the theoretical response is correct for a realistic value of 27 microns for $\delta^{f}$ in regards to the diameter of the particles (250 microns). The downturns of the stress-strain responses occur at similar values of macroscopic strain. Finally, one notes a satisfactory estimate of the material volume change upon stretching. On the downside, the initial stiffness of the material could not be reached. Increasing the cohesive zone stiffness slows down the simulations severely, ultimately favoring numerical divergences. Note that the calculation diverged before displaying a possible upturn. Nonetheless, such an upturn, controlled by the matrix behavior, was obtained for other cohesive zone parameters, driving unfortunately to unsatisfactory comparisons with the experiments.

\subsection{Additional remarks based on further numerical simulations}

Additional analyses have been carried out. The same simulations were run on microstructures defined by different distributions of spherical beads. Similar trends were obtained showing that the distribution of the particles in space has little impact on the results discussed here. Only the strain values at which damage appears or adhesion failure is reached, vary slightly.

We have discussed above the strong impacts of parameters $T^{c}$ and $\delta^{f}$ on the local damage and induced macroscopic behavior of the composite. In order to show explicitly the weak impact of the energy of adhesion $\Gamma$, which is an intrinsic parameter drawing usually significant interest, macroscopic stress-strain responses have been computed for two sets of interfacial properties presenting the same adhesion energy $\Gamma$. Fig. 12 shows the traction-separation behaviors considered and the resulting macroscopic stress-strain responses. Two very different types of local damage and resulting macroscopic behaviors were obtained driven by very different values of $T^{c}$ and $\delta^{f}$.

Finally, a more complex cohesive-zone model defined by Park et al. (2009) has been examined. A modified thermodynamically consistent version (Gilormini et al., 2017) has been implemented in Abaqus via a suitable UEL routine. This involved a smoother evolution of the traction separation law but to the cost of up to eight parameters when normal and tangent adhesion behaviors differ. The interest of such a complex potential-based approach is to bring more flexibility when quantitative comparisons are attempted. Despite the increased complexity of the cohesive law, the results were found similar in terms of the computed macroscopic behavior and the associated local damage features. Moreover, an attempt to do better in terms of quantitative representation of the actual material behavior was unsuccessful.

\section{Conclusion}

The macroscopic mechanical behavior of a rubberlike matrix highly filled with spherical micrometric and mono-size particles has been examined at the light of the damage that develops locally. An actual material was prepared in the lab and its mechanical response was characterized in uniaxial tension. X-ray tomography imaging has revealed the evolution of local damage upon deformation: debonding at 
the matrix/filler interfaces appears early at the poles of the particles, and its ensuing coalescence creates damage localization surfaces.

In order to understand the effects of the matrix/filler interfacial behavior, three-dimensional numerical materials have been generated for finite element analyses, where a simple version of the classic Tvergaard-Hutchinson cohesive-zone model has been used to model the damageable matrix/filler interfaces. While the numerical materials involved a relatively small periodic cell containing 64 beads in order to reduce the computational cost to a reasonable extent, all the trends observed experimentally were obtained, and several interesting results were reached:

- First, damage starts with matrix debonding at the poles of the particles and is distributed evenly across the microstructure. Then, some interfaces fail at one of the poles of some beads. These interface failures may be either localized in a plane perpendicular to the direction of stretching, or scattered.

- The distribution of the particles in the periodic cell has little impact on how damage evolves, whereas the effect of the behavior of the matrix/filler interface is of first order.

- The critical strength involved in the matrix/filler interface behavior determines the local distribution of severe damage. Damage localizes in specific areas of the microstructure for high values of the critical strength, whereas the particles showing complete interface failure are scattered for low values. Nonetheless, high values of the adhesion critical strength induce high material strength.

- Increasing the interface separation displacement to failure limits complete adhesion loss and preserves the integrity of the composite material.

\section{Declaration of Competing Interest}

The authors declare that they have no known competing financial interests or personal relationships that could have appeared to influence the work reported in this paper.

\section{Acknowledgements}

Acquisition of the microtomography device of laboratoire Navier (ENPC) has been made possible thanks to grants from the Région Ile-deFrance (SESAME 2007 program) and CNRS. This work was supported by the ANR under contract number ANR-10-EQPX-37, and the authors thank the Délégation Générale de l'Armement (DGA) and ArianeGroup, Vert-le-Petit (France), for their financial supports.

\section{References}

Abaqus, 2018. Abaqus Standard Version 2018. Dassault Systèmes Simulia Corp. Böhm, H.J., Han, W., Eckschlager, A., 2004. Multi-inclusion unit cell studies of reinforcement stresses and particle failure in discontinuously reinforced ductile matrix composites. CMES Comp. Model Eng. 5, 5-20.

Chaboche, J.L., Feyel, F., Monerie, Y., 2001. Interface debonding models: a viscous regularization with a limited rate dependency. Int. J. Solids Struct. 38, 3127-3160.

Cho, Y., Lee, W., Park, Y., 2017. Finite element modeling of tensile deformation behaviors of iron syntactic foam with hollow glass microspheres. Materials 10, 1201.

Cornwell, L.R., Schapery, R.A., 1975. SEM Study of microcracking in strained solid propellant. Metallography 8, 445-452.

Cras, J.J., Rowe-Taitt, C.A., Nivens, D.A., Ligler, F.S., 1999. Comparison of chemical cleaning methods of glass in preparation for silanization. Biosensors Bioelec. 14, 683-688.

Farris, R.J., 1968. The character of the stress-strain function for highly filled elastomers Trans. Soc. Rheol. 12, 303-314.

de Francqueville, F., Gilormini, P., Diani, J., 2019. Representative volume elements for the simulation of isotropic composites highly filled with monosized spheres. Int. J. Solids Struct. 158, 277-286.

Gao, Y.F., Bower, A.F., 2004. A simple technique for avoiding convergence problems in finite element simulations of crack nucleation and growth on cohesive interfaces. Model. Simul. Mater. Sci. Eng. 12, 453-463.

Gilormini, P., Toulemonde, P.A., Diani, J., Gardere, A., 2017. Stress-strain response and volume change of a highly filled rubbery composite: experimental measurements and numerical simulations. Mech. Mater. 111, 57-65.

Guo, A., Shi, X., Chen, Y., Chen, H., Peng, X., Harrison, P., 2014. Mechanical modeling of incompressible particle-reinforced neo-Hookean composites based on numerical homogenization. Mech. Mater. 70, 1-17.

Gusev, A., 2016. Controlled accuracy finite element estimates for the effective stiffness of composites with spherical inclusions. Int. J. Solids Struct. 80, 227-236.

Hamitouche, L., Tarfaoui, M., Vautrin, A., 2008. An interface debonding law subject to viscous regularization for avoiding instability: application to the delamination problems. Eng. Fract. Mech. 75, 3084-3100.

Inglis, H.M., Geubelle, P.H., Matouš, K., Tan, H., Huang, Y., 2007. Cohesive modeling of dewetting in particulate composites: micromechanics vs. multiscale finite element analysis. Mech. Mater. 39, 580-595.

Katzav, E., Berdichevsky, R., Schwartz, M., 2017. Random close packing and the hard sphere Percus-Yevick theory. arXiv:1703.09903.

Leonard, M., Wang, N., Lopez-Pamies, O., Nakamura, T., 2020. The nonlinear elastic response of filled elastomers: experiments vs. theory for the basic case of particulate fillers of micrometer size. J Mech. Phys. Solids 135, 103781.

Li, G., Wang, Y., Jiang, A., Yang, M., Li, J., 2018. Micromechanical investigation of debonding processes in composite solid propellants. Prop. Expl. Pyro. 43, 642-649.

Lopez-Pamies, O., Goudarzi, T., Danas, K., 2013. The nonlinear elastic response of suspensions of rigid inclusions in rubber: II-a simple explicit approximation for finiteconcentration suspensions. J Mech. Phys. Solids 61, 19-37.

Llorca, J., Segurado, J., 2004. Three-dimensional multiparticle cell simulations of deformation and damage in sphere-reinforced composites. Mater. Sci. Eng. A365, $267-274$.

Lubachevsky, B.D., Stillinger, F.H., 1990. Geometric properties of random disk packings. J. Stat. Phys. 60, 561-583.

Matouš, K., Geubelle, P.H., 2006. Finite element formulation for modeling particle debonding in reinforced elastomers subjected to finite deformations. Comp. Methods Appl. Mech. Eng. 196, 620-633.

Matouš, K., Inglis, H.M., Gu, X., Rypl, D., Jackson, T.L., Geubelle, P.H., 2007. Multiscale modeling of solid propellants: from particle packing to failure. Compos. Sci. Techno. 67, 1694-1708.

Moraleda, J., Segurado, J., Llorca, J., 2009. Effect of interface fracture on the tensile deformation of fiber-reinforced elastomers. Int. J. Solids. Struct. 46, 4287-4297.

Oberth, A.E., Bruenner, R.S., 1965. Tear phenomena around solid inclusions in castable elastomers. Trans. Soc. Rheol. 9, 165-185.

Ogden, R. W., 1982. Elastic deformations of rubberlike solids. mechanics of solids, the Rodney Hill 60th anniversary volume. Pergamon, 499-537.

Park, K., Paulino, G.H., 2011. Cohesive zone models: a critical review of traction-separation relationships across fracture surfaces. Appl. Mech. Rev. 64, 60802

Park, K., Paulino, G.H., Roesler, J.R., 2009. A unified potential-based cohesive model of mixed-mode fracture. J. Mech. Phys. Solids. 57, 891-908.

Schneider, K., Klusemann, B., Bargmann, S., 2016. Automatic three-dimensional geometry and mesh generation of periodic representative volume elements for matrixinclusion composites. Adv. Eng. Softw. 99, 177-188.

Segurado, J., Llorca, J., 2005. A computational micromechanics study of the effect of interface decohesion on the mechanical behavior of composites. Acta Mater. 53, 4931-4942.

Segurado, J., Llorca, J., 2002. A numerical approximation to the elastic properties of sphere-reinforced composites. Mech. Phys. Solids 50, 2107-2121.

Skoge, M., Donev, A., Stillinger, F.H., Torquato, S., 2006. Packing hyperspheres in highdimensional euclidean spaces. Phys. Rev. E 74, 041127.

Spring, D.W., Paulino, G.H., 2015. Computational homogenization of the debonding of particle reinforced composites: the role of interphases in interfaces. Comput. Mat. sci. 109, 209-224.

Tan, H., Liu, C., Huang, Y., Geubelle, P.H., 2005. The cohesive law for the particle/matrix interfaces in high explosives. J. Mech. Phys. Solids. 53, 1892-1917.

Torquato, S., 2002. Random Heterogeneous Materials: Microstructure and Macroscopic Properties. Springer-Verlag, New York.

Toulemonde, P.A., Diani, J., Gilormini, P., Desgardin, N., 2015. A numerical study of the influence of polydispersity on the behaviour until break of a reinforced hyperelastic material with a cohesive interface. Mater. Techn. 103, 306.

Toulemonde, P.A., Diani, J., Gilormini, P., Desgardin, N., 2016. On the account of a cohesive interface for modeling the behavior until break of highly filled elastomers. Mech. Mater. 93, 124-133.

Tvergaard, V., Hutchinson, J.W., 1993. The influence of plasticity on mixed mode interface toughness. J. Mech. Phys. Solids. 41, 1119-1135.

Zhang, B., Yiu, X., Gu, B., 2018. Modeling and experimental validation of interfacial fatigue damage in fiber-reinforced rubber composites. Polym. Eng. Sci. 58, 920-927. 